Article

\title{
Distinguishing Technical Inefficiency from Desirable and Undesirable Congestion with an Application to Regional Industries in China
}

\section{Jun Wang and Yong Zha *}

School of Management, University of Science and Technology of China, Hefei 230026, China; E-Mail: wangjun1200@mail.ustc.edu.cn

* Author to whom correspondence should be addressed; E-Mail: zhabeer@ustc.edu.cn; Tel.: +86-138-6672-4969; Fax: +86-551-360-0025.

External Editor: Panayiota Alevizou

Received: 11 September 2014; in revised form: 3 November 2014 / Accepted: 19 November 2014 / Published: 2 December 2014

\begin{abstract}
Congestion is an important issue that requires the efficiency of decision-making units (DMUs). We first classify conventional congestion into congestion (newly defined) and technical inefficiency, based on prior research and real applications. Modified definitions and mathematical expression of congestion, managerial inefficiency, and technical inefficiency are proposed to better illustrate the differences between them. Several modified models are provided to identify and recognize those types of inefficiencies and congestion. We then extend the model by considering the desirable and undesirable types of congestion simultaneously. The proposed approach is applied and verified by identifying resource congestion and environmental inefficiencies in China's economic development.
\end{abstract}

Keywords: data envelopment analysis; technical inefficiency; managerial inefficiency; desirable and undesirable congestion

\section{Introduction}

The rapid development of the economy has substantially increased energy demand and caused serious environmental problems in China [1]. Two key issues accompany the development and are suggested as major concerns. One is congestion. A greater consumption of resources cannot necessarily produce more output. It creates a poor economic state when firms invest excessive input in production units. 
Cooper et al. [2] describe the situation thusly: "congestion is said to when the output that is maximally possible can be increased by reducing one or more inputs without improving any other inputs or outputs. Conversely, congestion may occur when some outputs are reduced by increasing one or more inputs without improving any other inputs or outputs." Identifying and managing the factors which affect congestion is an intriguing aspect of the research in Data Envelopment Analysis (DEA). Many efforts have been made by researchers to explore whether congestion occurs and how its effects impose on productive and managerial practice. For example, Färe and Grosskopf [3] propose a DEA framework to quantitatively analyze the congestion. Sueyoshi [4] introduces the congestion issue in its dual equations and discusses the economic implications for production economics.

Another concern is the byproducts that accompany the consumption of energy. As a resource input, energy generates a lot of pollutants and damage to the environment during the production process. For example, Korhonen and Luptacik [5] treat emissions directly as input and suggest they should be decreased to measure the eco-efficiency of coal-fired power plants in a European country. Thus, outputs are suggested to better identify whether they are desirable. Specifically, when congestion is involved with the desirability of the outputs, it may be difficult to distinguish which input is responsible for the desirable or undesirable outputs. Sueyoshi and Goto [6] extend the concept of congestion by identifying its characteristics as either "desirable (good)" and "undesirable (bad)." Such a situation often occurs when the outputs are classified as desirable or undesirable. For example, desirable congestion can occur between the inputs and undesirable outputs. That is, undesirable outputs can be increased by decreasing one or more inputs while retaining any other inputs or outputs as constant. This type of congestion is desirable for managers. Based on the definition, Wu et al. [7] examine the situation where desirable and undesirable outputs coexist simultaneously.

Although previous research has attempted to illustrate the varying status of congestion and describe congestion in a concise and practical way, there has been little effort to make a distinct difference between inefficiency and congestion. For example, some researchers identify congestion as one type of inefficiency that affects performance and profits of firms severely in real practical applications [8], while others extend congestion to include some of those inefficiencies, which exaggerates the extent of congestion [9]. In order to better illustrate how inefficiencies differ from congestion, we take as an example an inefficient decision making unit (DMU), which we have an incentive to make efficient. Without loss of generality, we discuss the situation in an output orientation. If the inefficient inputs and outputs are projected onto the efficient frontier defined by [9], it needs three sequential projections: (1) hold the inputs constant and increase the outputs to reach the frontier; (2) decrease the inputs and increase the outputs while retaining the unit's efficiency, that is, having the unit move along the efficient frontier; and (3) hold the outputs constant and decrease the inputs while retaining the unit's efficiency, which implies that the unit also shifts along the frontier. According to [9], the first projection reflects managerial inefficiencies and the last two projections reflect congestion. Such classifications eliminate the distinction between congestion and inefficiency. Previous studies focusing on congestion do not make further comment on the issue. However, in related inefficiency studies, such classification may lead to confusion and is seen as paradoxical.

In order to remedy the confusion between inefficiency and congestion in the existing literature, we first introduce the concept of inefficiencies with different characteristics based on previous literature and propose a more precise definition of congestion. Based on the three projections as [10] describes, we 
class them as managerial inefficiency, congestion, and technical inefficiency, respectively. We also propose a mathematical formulation to illustrate inefficiencies and congestion. Then we propose modified models to separate technical inefficiency from congestion and analyze the properties that determine whether and how congestion and inefficiencies occur. Since undesirable output is accompanied by desirable output, it is necessary to incorporate additional considerations into existing DEA models so as to properly decrease the undesirable output and increase the desirable output. Thus, a new approach for measuring congestion with undesirable outputs is applied to analyzing congestion in industry in 31 administrative regions of China. We are trying to identify the factors which may restrict China's economic development and to make some policy proposals for the government.

The remainder of this article has the following structure: Section 2 provides a literature review. Section 3 briefly introduces the theoretical background of slack variables in DEA models and distinguishes technical inefficiency, managerial inefficiency, and congestion. We describe the mathematical concept of technical and managerial inefficiency, or congestion, more accurately. A modified DEA framework evaluating inefficiencies and congestion is proposed in Section 4. Section 5 proposes a method for identifying desirable and undesirable congestion. In Section 6, we present an empirical example to verify the feasibility of the proposed approach. Conclusions are made in the last section.

\section{Literature Review}

After the first paper that discussed congestion with a solid theoretical basis [11], many efforts have been made by researchers to explore whether congestion occurs and how congestion impacts on productive and managerial practices. Here we review the studies related to our paper; these can be classified into four categories: the first category studies the generation and effect of congestion; the second category discusses model development and the application of congestion measurements; the third category investigates the relationship between inefficiency and congestion; and the fourth considers undesirable problems in the DEA framework for measuring congestion.

Several papers have investigated the generation and effect of congestion. For example, Färe and Grosskopf [12] suggest a procedure for identifying those input factors that cause congestion. Cooper et al. [13] explore an integrative model to estimate congestion and identify the sources of congestion. Flegg [14] finds that the generation of congestion may be different for three alternative methods. Simões and Marques [15] determine and compare the influence of congestion on efficiency using different methodologies.

Some other papers highlight congestion measurement using different models. Khodabakhshi [16] proposes a one-model approach based on relaxed combination of inputs in order to simplify the calculation process. Noura et al. [17] study a new method which requires considerably less computation and proves equivalent to [13]. Khoveyni et al. [18] propose a slack-based DEA approach to identify congestion for the evaluated DMUs. Other papers study the application of the proposed model. Kao [19] applies the new model approach to measure the congestion effect of Taiwan's forests. Simões and Marques [20] apply three different methodologies to compare congestion measurement and evaluate the performance of Portuguese hospitals. The above literature studies focus on the identification and measurement of congestion and do not involve other kinds of inefficiencies.

Distinguishing between different kinds of inefficiencies can help managers make better use of resources to produce higher output and achieve optimal allocation of resources. Cooper et al. [2] 
investigate the management of congestion in Chinese industry, and discuss how elimination of managerial inefficiency can contribute to output augmentation without reducing employment in the textile and automobile industries. Cooper et al. [13] suggest that inefficiency is the necessary condition for the presence of congestion. Tone and Sahoo [9] propose the congestion-weak model to identify weak congestion, but their model cannot separate congestion from technical inefficiency. Brockett et al. [10] introduce a method for discussing congestion and technical inefficiency. They investigate how managerial inefficiencies are identified and calculated separately from the effect of congestion. Marques and Simões [21] use three different approaches to estimate the influence of congestion on the technical efficiency of the airport. Their work tells us how to reasonably consider managerial and technical inefficiencies. However, their studies do not distinguish technical inefficiency from congestion in the production processes. In addition, the above approaches only consider traditional scenarios, which ignore undesirable output.

There is a body of work dealing with the undesirable problem in DEA framework. For example, Färe et al. [22] propose a model to deal with the undesirable output problem. Hailu and Veeman [23] suggest treating undesirable output as input for the reason that the producer wishes to increase desirable output and decrease input and undesirable output. Seiford and Zhu [24] introduce a new method to deal with undesirable output via data transformation. Ji and Wang [25] apply Seiford's linear transformation method to evaluate efficiency with respect to the undesirable output. Jahanshahloo et al. [26] present an alternative method for dealing with undesirable input and output in non-radial DEA models. Huang et al. [27] consider undesirable output when measuring bank efficiency with a two-stage network model. Li et al. [28] also investigate desirable and undesirable outputs in evaluating the performance of electric power supply companies in China. In addition, the undesirable issues are extended to identify congestion based on output characteristics. Sueyoshi and Goto [6] classify the concept of congestion into "undesirable (bad) congestion" and "desirable (good) congestion." They also propose that Wu et al. [7] introduce a new approach for measuring congestion with undesirable outputs and discuss the congestion of the industry in 31 administrative regions of China.

Thus, with respect to the production of undesirable output, how to identify desirable and undesirable congestion, as well as make an appropriate input resources investment, is an urgent problem for firms today and in the future. Our current work differs from these previous papers in two main ways. First, whereas most of the existing literatures focus on congestion measurement, the bulk of our analysis is to make a differentiation between technical inefficiency, managerial inefficiency, and congestion. In particular, we show how to project the inefficient units into the efficient frontier by eliminating the inefficiencies given the existence of congestion. Second, we extend the conventional congestion concept to encompass undesirable and desirable congestion when undesirable output is included.

\section{Definition}

\subsection{A Numerical Example}

As illustrated in Figure 1, initiated by [10], six points help to make a clear distinction between technical inefficiency, congestion, and managerial inefficiency. For simplicity's sake, a unit is assumed to consume a single input to produce a single output. The value on the left in any of the parentheses corresponds to an input amount and that on the right corresponds to an output amount. Thus, $(1,0.5)$ 
alongside point A means that DMU A utilizes one unit of input to produce half a unit of output. The frontier is composed of a piecewise segment with the points ABCD. Any inefficient unit is suggested to move along the frontier via a typical path depicted as follows. We take point $\mathrm{E}$ as an example. First, hold the input constant and increase the output to reach the frontier; second, move along the frontier as the input is decreased and the output is increased simultaneously; third, hold the output constant and decrease the input along the frontier. Following these three steps, point $\mathrm{E}$ is projected to E', C, and B sequentially. In [9], the projection from E' to B is defined as the congestion. However, that may be not the case. In the third step, although the input decreases, the output does not increase, which does not accord with the definition of congestion [13]. Next, we will make definitions of inefficiencies and congestion according to the projections and three steps.

Figure 1. Example.

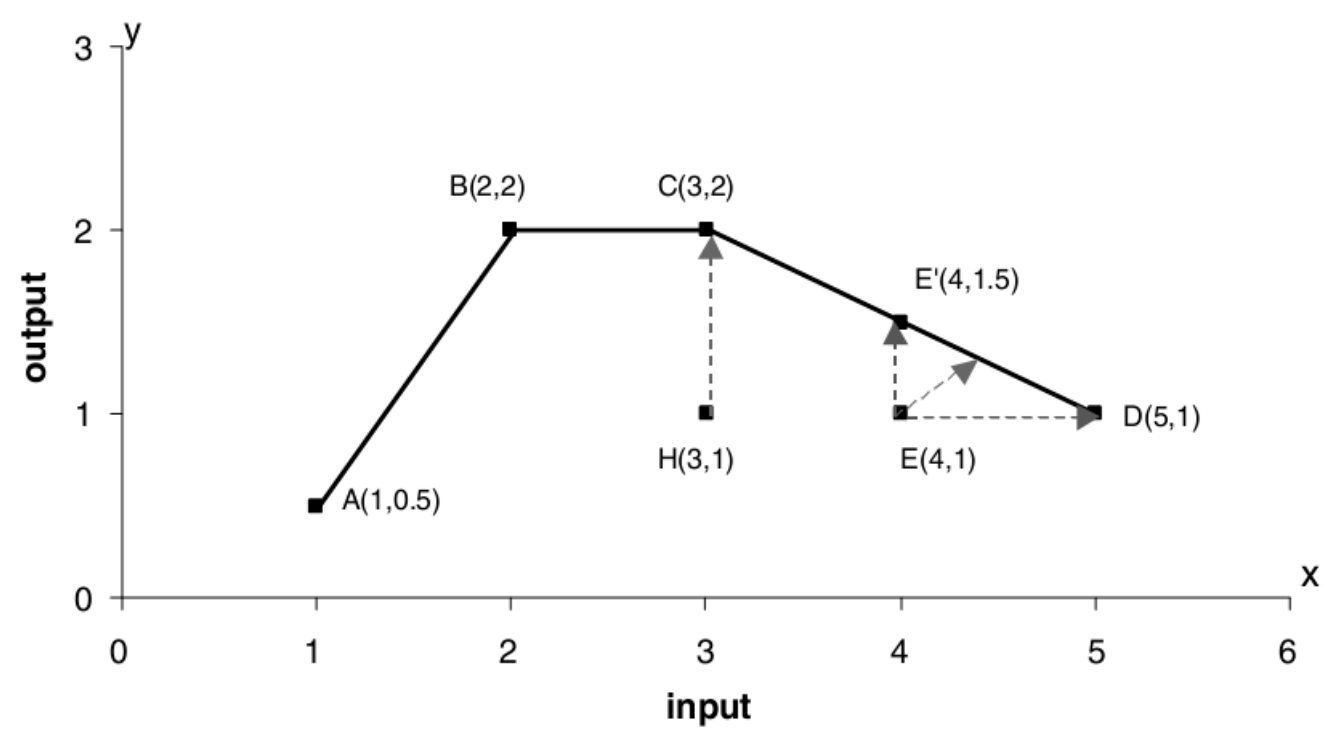

Based on the above analysis and the definitions in literature, we divide congestion in [9] into two parts, technical inefficiency and congestion. In [2,10], managerial inefficiency is defined as the output augmentation without reducing input, which corresponds to the move from $\mathrm{E}$ to $\mathrm{E}$ ' in Figure 1 (the first projection). The second projection displays an input reduction and an output increase. It is defined as congestion, which corresponds to the move from E' to C. According to [10], technical inefficiency is defined as a decrease of the input without increasing the output, which corresponds to the move from $\mathrm{C}$ to $\mathrm{B}$.

\subsection{Mathematical Expression of Technical Inefficiency, Managerial Inefficiency, and Congestion}

In congestion-related literature, the definitions of inefficiency and congestion lack a formal and unified mathematical expression. In particular, some of them are not accurate and may be paradoxical. In order to better describe congestion and inefficiencies, we give mathematical representations to illustrate them in a precise way.

Take $n$ observed DMUs, each of which consumes $m$ inputs and produce $s$ outputs. The input and output variables of $D M U_{j}$ are $x_{i j}(i=1, \ldots, m)$ and $y_{i j}(r=1, \ldots, s)$, respectively. For simplicity's sake, we denote vectors $x_{j}$ and $y_{j}$ as the vectors of the inputs and outputs of $D M U_{j}$, and $X$ and $Y$ as the matrix of 
the inputs and outputs of the DMUs, respectively. The new production possibility set $S_{N E W}$ related to the input-output correspondence is formulated as:

$$
S_{N E W}=\{(x, y) \mid x=\lambda X, y \leq \lambda Y, \lambda e=1, \lambda \geq 0\} .
$$

Therefore, the mathematical definition of congestion and the two types of inefficiency are as follows:

Managerial inefficiency: For any given $D M U_{0}\left(x_{0}, y_{0}\right)$, if there exists a $D M U_{0}^{M}\left(x_{0}^{M}, y_{0}^{M}\right)$ on the $S_{N E W}$ frontier which satisfies $x_{0}^{M}=x_{0}$ and $y_{0}^{M} \geq y_{0}$, managerial inefficiency occurs and is measured as $\max \left(y_{0}^{M}-y_{0}\right)$.

Congestion: For any given $D M U_{0}^{M}\left(x_{0}^{M}, y_{0}^{M}\right)$, which is derived from $D M U_{0}\left(x_{0}, y_{0}\right)$ and has managerial inefficiency excluded preliminarily, if there exists a new $D M U_{0}^{C}\left(x_{0}^{C}, y_{0}^{C}\right)$ on the $S_{N E W}$ frontier such that $y_{0}^{C} \geq y_{0}^{M}$ and $x_{0}^{C} \leq x_{0}^{M}$, congestion occurs and is measured according to the following formula:

(1) Output deficiency: $\max \left(y_{0}^{C}-y_{0}^{M}\right)$;

(2) Input redundancy: $\min \left(x_{0}^{C}-x_{0}^{M}\right)$ under the condition of $\max \left(y_{0}^{C}-y_{0}^{M}\right)$.

Technical inefficiency: For any given $D M U_{0}^{C}\left(x_{0}^{C}, y_{0}^{C}\right)$, which is derived from $D M U_{0}\left(x_{0}, y_{0}\right)$ and has managerial inefficiency and congestion excluded preliminarily, if there exists a $D M U_{0}^{T}\left(x_{0}^{T}, y_{0}^{T}\right)$ on the $S_{N E W}$ frontier such that $x_{0}^{T} \leq x_{0}^{C}$ and $y_{0}^{T}=y_{0}^{C}$, technical inefficiency occurs and is measured as $\max \left(x_{0}^{T}-x_{0}^{C}\right)$.

Thus, we can use the above definitions to estimate the inefficiencies and congestion of an inefficient DMU, and project it onto the efficient frontier by eliminating managerial inefficiency, congestion, and technical inefficiency, respectively. It is worth noting that the definitions are given under the condition that the outputs are desirable for the decision makers; we then extend them by incorporating different classifications of outputs. That is, desirable and undesirable outputs are taken into consideration simultaneously and congestion is divided between desirable congestion and undesirable congestion.

\section{Identifying Inefficiencies and Congestion}

In this section, we first identify managerial inefficiency and then classify congestion and technical inefficiency based on the definition proposed by [9]. For simplicity's sake, we do not take undesirable outputs into consideration.

\subsection{Identifying Managerial Inefficiency}

Banker et al. [29] introduce the BCC model to estimate the efficiency of an evaluated DMU under the variable returns to scale setting: 
$\max \theta$

$$
\begin{array}{ll}
\text { s.t. } & x_{i 0}=\sum_{j=1}^{n} \lambda_{j} x_{i j}+s_{i}^{-}, i=1, \ldots, m \\
& \theta y_{r 0}=\sum_{j=1}^{n} \lambda_{j} y_{r j}-s_{r}^{+}, r=1, \ldots, s \\
& \sum_{j=1}^{n} \lambda_{j}=1 \\
& \lambda_{j} \geq 0, s_{i}^{-}, s_{r}^{+} \geq 0 \text { for } \forall i, j, r .
\end{array}
$$

In order to identify to what extent the output can be increased maximally, and to estimate managerial inefficiency of the evaluated DMU, similar to the BCC model, we project all the DMUs onto the $S_{N E W}$ frontier using the following model:

$\max \theta$

$$
\begin{array}{ll}
\text { s.t. } & x_{i 0}=\sum_{j=1}^{n} \lambda_{j} x_{i j}, i=1, \ldots, m \\
& \theta y_{r 0}=\sum_{j=1}^{n} \lambda_{j} y_{r j}-s_{r}^{M}, r=1, \ldots, s \\
& \sum_{j=1}^{n} \lambda_{j}=1 \\
& \lambda_{j} \geq 0, s_{r}^{M} \geq 0 \text { for } \forall j, r .
\end{array}
$$

It is worth noting that the first constraint of Model (3) differs from the BCC model in that the slacks of inputs are ignored. In this way, we can keep $x$ unchanged so as to investigate managerial inefficiency. If $D M U_{0}\left(x_{0}, y_{0}\right)$ is inefficient and is not on the frontier $S_{N E W}$, we project it through Equations (4) and (5).

$$
\begin{gathered}
x_{i 0}^{M}=x_{i 0}, i=1, \ldots, m \\
y_{r 0}^{M}=\theta^{*} y_{r 0}+s_{r}^{M^{*}}, r=1, \ldots, s,
\end{gathered}
$$

where $\left(\theta^{*}, s_{r}^{M^{*}}\right)$ are the optimal solutions of Model (3). Following the equation, $x_{i 0}$ remains unchanged, and $y_{r 0}$ increases according to the corresponding proportion and slack of Equation (5). Thus, the new input-output vector $\left(x_{0}^{M}, y_{0}^{M}\right)$ is on the frontier $S_{N E W}$. Based on the characteristics of managerial inefficiency, we can obtain the following property:

Property 1. If one or more of the slacks $s_{r}^{M^{*}} \neq 0$ for the evaluated $D M U_{0}$, managerial inefficiency occurs and is measured by $y_{r 0}^{M}-y_{r 0}$ for output $r$.

\subsection{Identifying Congestion and Technical Inefficiency}

With the above transformation, $\left(x_{0}, y_{0}\right)$ is converted to $\left(x_{0}^{M}, y_{0}^{M}\right)$, and the evaluated $D M U_{0}$ is projected onto the frontier $S_{N E W}$. Obviously, managerial inefficiency is extracted from $D M U_{0}$. Based on the new $D M U_{0}^{M}\left(x_{0}^{M}, y_{0}^{M}\right)$, we begin with the model of [9] and classify the congestion of [9] into congestion and technical inefficiency: 


$$
\begin{array}{ll}
\max & \frac{1}{s} \sum_{r=1}^{s} \frac{s_{r}^{C}}{y_{r 0}^{M}}+\varepsilon \frac{1}{m} \sum_{i=1}^{m} \frac{s_{i}^{T-}}{x_{i 0}^{M}} \\
\text { s.t. } & x_{i 0}^{M}=x_{j}^{M} \lambda_{j}+s_{i}^{T-}, i=1, \ldots, m \\
& y_{r 0}^{M}=y_{j}^{M} \lambda_{j}-s_{r}^{C}, r=1, \ldots s \\
& \sum_{j=1}^{n} \lambda_{j}=1 \\
& \lambda_{j} \geq 0, s_{i}^{T-}, s_{r}^{C} \geq 0 \text { for } \forall i, j, r .
\end{array}
$$

The objective of Model (6) is to maximize the slacks of the outputs as a first priority. Given that the outputs are optimized, Model (6) allows us to maximize the slacks of the inputs as a secondary priority. Denote $\left(\lambda^{*}, s^{T-*}, s^{C^{*}}\right)$ as the optimal vector of Model (6). Tone and Sahoo [9] illustrate that $D M U_{0}^{M}$ is congested if and only if $s^{C^{*}} \neq 0$, as derived from Model (6). Specifically, congestion is measured by $s^{T-*}$ and $s^{C^{*}}$ as the input redundancy and output shortage, respectively. The projected inputs and outputs are obtained from:

$$
\begin{aligned}
& \hat{x}_{i 0}=x_{i 0}^{M}-s_{i}^{T-*}, i=1, \ldots, m \\
& \hat{y}_{r 0}=y_{r 0}^{M}+s_{r}^{C^{*}}, r=1, \ldots, s .
\end{aligned}
$$

However, in real applications, this is not the case. We take the following data analysis as an illustration. We apply the data of Figure 1 and list them in Table 1.

Table 1. The difference between inefficiency and congestion.

\begin{tabular}{cccccccc}
\hline \multirow{2}{*}{ DMU } & \multirow{2}{*}{$\boldsymbol{x}$} & $\boldsymbol{y}$ & $\boldsymbol{y}^{*}$ & $\hat{x}$ & $\hat{y}$ & $\boldsymbol{x}^{\boldsymbol{c}}$ & $\boldsymbol{y}^{\boldsymbol{c}}$ \\
\cline { 4 - 7 } & & & Model (5) & Models (7) and (8) & Models (10) and (11) \\
\hline $\mathrm{A}$ & 1 & 0.5 & 0.5 & 1 & 0.5 & 1 & 0.5 \\
$\mathrm{~B}$ & 2 & 2 & 2 & 2 & 2 & 2 & 2 \\
$\mathrm{C}$ & 3 & 2 & 2 & 2 & 2 & 3 & 2 \\
$\mathrm{D}$ & 5 & 1 & 1 & 2 & 2 & 3 & 2 \\
$\mathrm{E}$ & 4 & 1 & 1.5 & 2 & 2 & 3 & 2 \\
$\mathrm{H}$ & 3 & 1 & 2 & 2 & 2 & 3 & 2 \\
\hline
\end{tabular}

In Table 1, the second and third columns represent the original input and output of the units. The fourth column is obtained from Equation (5) and identifies the managerial inefficiency of the units. From Model (6), all the points are projected onto the frontier point B. This implies that the congestion contains the line segment BC. However, although point $\mathrm{C}$ has 1 unit in excess of the input, there is no output reduction associated with the input excess. According to [10], the line segment BC can represent the technical inefficiency, as defined above, instead of congestion.

In an effort to better distinguish technical inefficiency from congestion, we propose a modified model as follows: 


$$
\begin{array}{ll}
\max & \frac{1}{s} \sum_{r=1}^{s} \frac{s_{r}^{C}}{y_{r 0}^{M}}-\varepsilon \frac{1}{m} \sum_{i=1}^{m} \frac{s_{i}^{C-}}{x_{i 0}} \\
\text { s.t. } & x_{i 0}=x_{j}^{M} \lambda_{j}+s_{i}^{C-}, i=1, \ldots, m \\
& y_{r 0}^{M}=y_{j}^{M} \lambda_{j}-s_{r}^{C}, r=1, \ldots s \\
& \sum_{j=1}^{n} \lambda_{j}=1 \\
& \lambda_{j} \geq 0, s_{i}^{C-}, s_{r}^{C} \geq 0 \text { for } \forall i, j, r .
\end{array}
$$

Denote $\left(\lambda^{*}, s^{C-*}, s^{C^{*}}\right)$ as the optimal solution vector of Model (9). The only difference between Models (9) and (6) lies in that Model (9)'s secondary objective is to minimize the slacks of the inputs given that the slacks of outputs are maximized preliminarily. That is, in Model (9), the objective is to maximize the outputs first and then to minimize the inputs while retaining the first objective value of $\sum_{r=1}^{s} \frac{s_{r}^{C^{*}}}{y_{r 0}^{M}}$ at the optimum level. Similar to Equations (7) and (8), we can obtain the projected inputs and outputs as:

$$
\begin{gathered}
x_{i 0}^{C}=x_{i 0}^{M}-s_{i}^{C-*}, i=1, \ldots, m \\
y_{r 0}^{C}=y_{r 0}^{M}+s_{r}^{C^{*}}, r=1, \ldots, s .
\end{gathered}
$$

In this way, technical inefficiency is excluded from the congestion. According to the transformation, we can project all the points with congestion to point $\mathrm{C}$, and the congestion can be identified and estimated by the following properties:

Property 2. If one or more of the slacks $s_{i}^{C-*} \neq 0, i=1, \ldots, m$ and $s_{r}^{C^{*}} \neq 0, r=1, \ldots, s$ for the evaluated $D M U_{0}^{M}\left(x_{0}^{M}, y_{0}^{M}\right)$, congestion occurs. Specifically, if $s^{C-*} \neq 0$, input redundancy occurs and is measured by $x_{0}^{C}-x_{0}^{M}$ or $s^{C-*}$. If $s^{C^{*}} \neq 0$, output shortage occurs and is measured by $y_{0}^{C}-y_{0}^{M}$ or $S^{C^{*}}$.

Property 3. If $x_{0}^{C} \neq \hat{x}_{0}$ for the evaluated $\operatorname{DMU}_{0}^{M}\left(x_{0}^{M}, y_{0}^{M}\right)$, technical inefficiency occurs and the input redundancy is measured by $x_{0}^{C}-\hat{x}_{0}$.

Since $D M U_{0}\left(\hat{x}_{0}, \hat{y}_{0}\right)$ and $D M U_{0}\left(x_{0}^{C}, y_{0}^{C}\right)$ are the improvements derived from Models (7), (8), (10), and (11), respectively, we can identify technical inefficiency and congestion and distinguish them in a precise way. The results are clearly illustrated by the last two columns in Table 1 .

\section{Identifying Desirable and Undesirable Congestion}

In the previous section, we proposed several models for identifying managerial inefficiency and divided congestion as defined by [9] into congestion and technical inefficiency based on the related literature and real applications. However, this model cannot depict a situation where undesirable outputs do exist. As [6] suggested, desirable and undesirable congestion exist when the outputs are classified as desirable and undesirable. Specifically, desirable congestion can occur between inputs and undesirable outputs, whereas undesirable congestion occurs between inputs and desirable outputs. When some of the undesirable outputs decrease while other outputs remain constant when some of the inputs increase, desirable congestion occurs. Such a situation is desirable for decision makers if the marginal cost is 
lower than the marginal loss derived from undesirable outputs. With such an incentive, we extend the above models to illustrate a situation in which the undesirable outputs are considered simultaneously.

We begin with the definition of desirable congestion and then propose modified models to incorporate undesirable output and desirable congestion to better identify congestion that is preferable to the decision maker.

Denote the desirable and undesirable outputs as $g_{r j}(r=1, \ldots s)$ and $u_{t j}(t=1, \ldots q)$, respectively, and $u_{j}$ as the vector of the undesirable outputs of $D M U_{j}$. The definition of desirable congestion is as follows:

Desirable congestion: For any given $D_{M} U_{0}^{M}\left(x_{0}^{M}, g_{0}^{M}, u_{0}^{M}\right)$, which is derived from $D M U_{0}\left(x_{0}, g_{0}, u_{0}\right)$ when managerial inefficiency is excluded preliminarily, if there exists a new $D M U_{0}^{C}\left(x_{0}^{C}, g_{0}^{C}, u_{0}^{C}\right)$ on the $S_{N E W}$ frontier such that $u_{0}^{C} \geq u_{0}^{M}$ and $x_{0}^{C} \leq x_{0}^{M}$, desirable congestion occurs and is measured according to the following formula:

(1) Output deficiency: $\max \left(u_{0}^{C}-u_{0}^{M}\right)$;

(2) Input redundancy: $\min \left(x_{0}^{C}-x_{0}^{M}\right)$ under the condition of $\max \left(u_{0}^{C}-u_{0}^{M}\right)$.

We reformulate Model (9) as follows:

$$
\begin{array}{ll}
\max & \frac{1}{s} \sum_{r=1}^{s} \frac{s_{r}^{U}}{g_{r 0}^{M}}+\frac{1}{q} \sum_{t=1}^{q} \frac{s_{t}^{D}}{u_{t 0}^{M}}-\varepsilon \frac{1}{m} \sum_{i=1}^{m} \frac{s_{i}^{C-}}{x_{i 0}^{M}} \\
\text { s.t. } & x_{i 0}^{M}=x_{j}^{M} \lambda_{j}+s_{i}^{C-}, i=1, \ldots, m \\
& g_{r 0}^{M}=g_{j}^{M} \lambda_{j}-s_{r}^{U}, r=1, \ldots s \\
& u_{t 0}^{M}=u_{j}^{M} \lambda_{j}-s_{t}^{D}, t=1, \ldots, q \\
& \sum_{j=1}^{n} \lambda_{j}=1 \\
& \lambda_{j} \geq 0, s_{i}^{C-}, s_{r}^{U}, s_{t}^{D} \geq 0 \text { for } \forall i, j, r, t .
\end{array}
$$

where $s_{r}^{U}$ and $s_{t}^{D}$ are the slacks of desirable output $r$ and undesirable output $t$, respectively. Since desirable/undesirable output shortage is related to undesirable/desirable congestion, we use the superscripts " $U$ " and " $D$ " to denote undesirable and desirable congestion. Obviously, undesirable outputs are taken into consideration and are incorporated into Model (12). Compared with Model (9), Model (12) extends the objective by maximizing the slacks of desirable and undesirable outputs simultaneously; also, an additional constraint $u_{t 0}^{M}=u_{j}^{M} \lambda_{j}-s_{t}^{D}$ is added. It is worth noting that $u_{j}^{M}$ has been converted using a transformation similar to Model (5).

In real applications, as the input increases, both desirable outputs and undesirable outputs may decrease simultaneously. Thus, undesirable congestion occurs when some of the desirable outputs decrease, whereas desirable congestion occurs when some of the undesirable outputs decrease. This definition is different from [7] in that a reduction in input results in an increase in desirable outputs and decrease in undesirable outputs simultaneously.

We also utilize a two-stage process similar to [9] to solve Model (13). In the first stage, the slacks of desirable outputs and undesirable outputs are maximized simultaneously. That is, the first two parts of the objective function denoted by $O 1=\frac{1}{s} \sum_{r=1}^{s} \frac{s_{r}^{U}}{g_{r 0}^{M}}+\frac{1}{q} \sum_{t=1}^{q} \frac{s_{t}^{D}}{u_{t 0}^{M}}$ are maximized. In the second stage, $O 2=\frac{1}{m} \sum_{i=1}^{m} \frac{s_{i}^{C-}}{x_{i 0}^{M}}$ is minimized based on the objective value of the $O 1$ at the first stage. 
Thus, we can obtain the optimal solution vector $\left(\lambda^{*}, s^{C-*}, s^{U^{*}}, s^{D^{*}}\right)$ and project the original inputs and outputs as follows:

$$
\begin{aligned}
& x_{i 0}^{C}=x_{i 0}^{M}-s_{i}^{C-*}, i=1, \ldots, m \\
& g_{r 0}^{C}=g_{r 0}^{M}+s_{r}^{U^{*}}, r=1, \ldots, s \\
& u_{t 0}^{C}=u_{t 0}^{M}+s_{t}^{D^{*}}, t=1, \ldots, q .
\end{aligned}
$$

Based on the optimal solution above, we propose the following two properties:

Property 4. If $s^{U^{*}} \neq 0$ or $s^{D^{*}} \neq 0$ in the concerned $D M U_{0}^{M}\left(x_{0}^{M}, g_{0}^{M}, u_{0}^{M}\right)$, and $s^{C-*} \neq 0$, congestion is observed in the $D M U_{0}^{M}$. Undesirable congestion occurs if $s^{U^{*}} \neq 0$, whereas desirable congestion occurs if $s^{D^{*}} \neq 0$. Specifically, both desirable and undesirable congestion exist simultaneously if $s^{U^{*}} \neq 0$ and $s^{D^{*}} \neq 0$.

Property 5. If $s^{U^{*}}=0$ and $s^{D^{*}}=0$ in the concerned $D M U_{0}^{M}\left(x_{0}^{M}, g_{0}^{M}, u_{0}^{M}\right)$, and $s^{C-*}=0$, no congestion is observed in the $D M U_{0}^{M}$.

In an effort to better examine technical inefficiency, Model (6) is extended to consider undesirable outputs simultaneously:

$$
\begin{array}{ll}
\max & \frac{1}{s} \sum_{r=1}^{s} \frac{s_{r}^{U}}{g_{r 0}^{M}}+\frac{1}{q} \sum_{t=1}^{q} \frac{s_{t}^{D}}{u_{t 0}^{M}}+\varepsilon \frac{1}{m} \sum_{i=1}^{m} \frac{s_{i}^{T-}}{x_{i 0}^{M}} \\
\text { s.t. } & x_{i 0}^{M}=x_{j}^{M} \lambda_{j}+s_{i}^{T-}, i=1, \ldots, m \\
& g_{r 0}^{M}=g_{j}^{M} \lambda_{j}-s_{r}^{U}, r=1, \ldots s \\
& u_{t 0}^{M}=u_{j}^{M} \lambda_{j}-s_{t}^{D}, t=1, \ldots, q \\
& \sum_{j=1}^{n} \lambda_{j}=1 \\
& \lambda_{j} \geq 0, s_{i}^{T-}, s_{r}^{U}, s_{t}^{D} \geq 0 \text { for } \forall i, j, r, t .
\end{array}
$$

Denote the optimal solution vector of Model (16) as $\left(s^{T-*}, s^{U^{*}}, s^{D^{*}}\right)$, and the projected inputs and outputs are obtained as:

$$
\begin{aligned}
& \hat{x}_{i 0}=x_{i 0}^{M}-s_{i}^{T-*}, i=1, \ldots, m \\
& \hat{g}_{r 0}=g_{r 0}^{M}+s_{r}^{U^{*}}, r=1, \ldots, s \\
& \hat{u}_{r 0}=u_{r j}^{M^{*}}+s_{t}^{D^{*}}, t=1, \ldots, q .
\end{aligned}
$$

According to Property 3 , technical inefficiency can be distinguished from congestion by subtracting $\hat{x}_{j}$ from $x_{j}^{C}$. In addition, input congestion is estimated as $x_{j}^{M}-x_{j}^{C}$.

\section{Numerical Analysis}

In an effort to validate the feasibility of the proposed models in this study, we apply them to identifying resource congestion and environmental inefficiencies in China's economic development. 


\subsection{Data Description}

We selected data from 31 provinces, autonomous regions, and municipalities in mainland China for analysis. The inputs include total investment in fixed assets of industry $\left(x_{1}\right)$ and electricity consumption by industry $\left(x_{2}\right)$. The desirable output is gross industrial output value $(y)$ and undesirable outputs are total volume of waste water discharge $\left(u_{1}\right)$ and main pollutants emission in waste gas $\left(u_{2}\right)$. The main pollutants emitted include sulfur dioxide, nitrogen oxides, smoke, and dust, which are caused by industries. The two inputs refer to the fixed assets of the industry plus related fees, and the electricity consumption of the production processes. The gross industrial output is the most critical and representative output for a country. In real production processes, undesirable outputs are always accompanied by desirable outputs. We select waste water discharge and waste gas emission as the undesirable outputs that are regarded as two kinds of common waste. The data were collected from the China Statistical Yearbook 2013 [30] (see the Appendix for original data) and descriptions of the summary statistics are shown in Table 2.

Table 2. Statistical description of original data (sample size: 31 ).

\begin{tabular}{cccccc}
\hline Variables & & Mean & $\begin{array}{c}\text { Standard } \\
\text { Deviation }\end{array}$ & Minimum & Maximum \\
\hline \multirow{2}{*}{ Input } & $x_{1}$ & 9239.42 & 6154.69 & 424.68 & $25,207.36$ \\
& $x_{2}$ & 1599.72 & 1172.08 & 27.76 & 4619.41 \\
\hline Desirable Output & $g$ & $24,787.78$ & $20,705.85$ & 506.86 & $84,550.41$ \\
\hline \multirow{2}{*}{ Undesirable Output } & $u_{1}$ & $220,890.71$ & $181,034.33$ & 4683.14 & $838,550.53$ \\
& $u_{2}$ & 183.59 & 114.87 & 5.51 & 433.82 \\
\hline
\end{tabular}

\subsection{Analysis}

Table 3 represents the managerial inefficiency and congestion results when desirable and undesirable outputs are considered simultaneously. Columns 2-4 show the output deficiency caused by managerial inefficiency and slack variables obtained from Model (3). Several provinces exhibit managerial inefficiency, such as Tianjin, Inner Mongolia, Zhejiang, Fujian, Hainan, Chongqing, Sichuan, Guizhou, Yunnan, and Gansu. Hence, without altering the fixed assets and electricity utilized, the gross industrial output value (desirable output), and waste water and waste gas (undesirable outputs), are improved significantly. Our results also show that the output improvement in 2013 is even larger than the corresponding current output level in some districts. In fact, undesirable output deficiency caused by managerial inefficiency is desirable for decision makers. In any event, it indicates that significant shortfalls in output are due to managerial inefficiencies and removal of these inefficiencies can improve output without changing the resources.

Columns 5-9 report the output deficiency and input redundancy caused by congestion and slack variables obtained from Equation (12). This indicates that a decrease in some inputs may result in an increase in one or more outputs without worsening other inputs or outputs. Table 3 illustrates that Inner Mongolia, Liaoning, Anhui, Qinghai have congestion. The results show that input congestion occurs if and only if there are output congestions, which is illustrated by Property 2 . The decision maker can make a determination about the production process according to the occurrence of congestion. For example, 
desirable congestion happens in Liaoning province. This implies that a decrease in the input results in an increase in the undesirable output without worsening other inputs or outputs. It is a useful signal that decision makers can increase input continuously. In the opposite situation, the decision maker will maintain or reduce the original input in order to reduce undesirable congestion, as in Anhui province, which shows only undesirable congestion occurring. In a more complicated situation where desirable and undesirable congestion occur simultaneously, a decision maker would have to obtain more information to make decisions about production.

Table 3. Managerial inefficiency and congestion.

\begin{tabular}{|c|c|c|c|c|c|c|c|c|}
\hline \multirow{3}{*}{ District } & \multirow{2}{*}{\multicolumn{3}{|c|}{$\begin{array}{l}\text { Output improvement caused by } \\
\text { managerial inefficiency }\end{array}$}} & \multicolumn{5}{|c|}{ Congestion } \\
\hline & & & & \multicolumn{3}{|c|}{ Output shortage } & \multicolumn{2}{|c|}{$\begin{array}{c}\text { Input } \\
\text { redundancy }\end{array}$} \\
\hline & $y$ & $u_{1}$ & $u_{2}$ & $s_{1}^{U}$ & $s_{1}^{D}$ & $s_{2}^{D}$ & $s_{1}^{C-}$ & $s_{2}^{C-}$ \\
\hline Beijing & 0 & 0 & 0 & 0 & 0 & 0 & 0 & 0 \\
\hline Tianjin & 870 & 39,866 & 3 & 0 & 0 & 0 & 0 & 0 \\
\hline Hebei & 0 & 0 & 0 & 0 & 0 & 0 & 0 & 0 \\
\hline Shanxi & 0 & 0 & 0 & 0 & 0 & 0 & 0 & 0 \\
\hline Inner Mongolia & 5620 & 69,318 & 7 & 2331 & 2082 & 0 & 177 & 0 \\
\hline Liaoning & 0 & 0 & 0 & 0 & 7843 & 0 & 3672 & 0 \\
\hline Jilin & 0 & 0 & 0 & 0 & 0 & 0 & 0 & 0 \\
\hline Heilongjiang & 0 & 0 & 0 & 0 & 0 & 0 & 0 & 0 \\
\hline Shanghai & 0 & 0 & 0 & 0 & 0 & 0 & 0 & 0 \\
\hline Jiangsu & 0 & 0 & 0 & 0 & 0 & 0 & 0 & 0 \\
\hline Zhejiang & 3793 & 82,070 & 12 & 0 & 0 & 0 & 0 & 0 \\
\hline Anhui & 0 & 0 & 0 & 409 & 0 & 0 & 2133 & 0 \\
\hline Fujian & 5174 & 62,004 & 29 & 0 & 0 & 0 & 0 & 0 \\
\hline Jiangxi & 0 & 0 & 0 & 0 & 0 & 0 & 0 & 0 \\
\hline Shandong & 0 & 0 & 0 & 0 & 0 & 0 & 0 & 0 \\
\hline Henan & 0 & 0 & 0 & 0 & 0 & 0 & 0 & 0 \\
\hline Hubei & 0 & 0 & 0 & 0 & 0 & 0 & 0 & 0 \\
\hline Hunan & 0 & 0 & 0 & 0 & 0 & 0 & 0 & 0 \\
\hline Guangdong & 0 & 0 & 0 & 0 & 0 & 0 & 0 & 0 \\
\hline Guangxi & 0 & 0 & 0 & 0 & 0 & 0 & 0 & 0 \\
\hline Hainan & 440 & 6828 & 10 & 0 & 0 & 0 & 0 & 0 \\
\hline Chongqing & 1588 & 18,928 & 16 & 0 & 0 & 0 & 0 & 0 \\
\hline Sichuan & 3045 & 28,448 & 18 & 0 & 0 & 0 & 0 & 0 \\
\hline Guizhou & 6725 & 1917 & 4 & 0 & 0 & 0 & 0 & 0 \\
\hline Yunnan & 6780 & 30,073 & 31 & 0 & 0 & 0 & 0 & 0 \\
\hline Tibet & 0 & 0 & 0 & 0 & 0 & 0 & 0 & 0 \\
\hline Shaanxi & 0 & 0 & 0 & 0 & 0 & 0 & 0 & 0 \\
\hline Gansu & 5205 & 28,222 & 56 & 0 & 0 & 0 & 0 & 0 \\
\hline Qinghai & 0 & 0 & 0 & 3514 & 32,983 & 0 & 0 & 137 \\
\hline Ningxia & 0 & 0 & 0 & 0 & 0 & 0 & 0 & 0 \\
\hline Xinjiang & 0 & 0 & 0 & 0 & 0 & 0 & 0 & 0 \\
\hline
\end{tabular}


Tables 4 and 5 show the new desirable and undesirable outputs of the DMUs after eliminating managerial inefficiency and congestion, respectively. Managerial inefficiency illustrates that the output can increase without a change in the input; congestion suggests that the output may increase with the decrease of the input. Comparing the results of the two tables below, we find that both managerial inefficiency and congestion occur in Inner Mongolia, which can be projected into the efficient frontier by eliminating managerial inefficiency and congestion simultaneously.

Table 4. Output improvement after eliminating managerial inefficiency.

\begin{tabular}{cccc}
\hline District & $\boldsymbol{g}^{\boldsymbol{M}}$ & $\boldsymbol{u}_{1}^{M}$ & $\boldsymbol{u}_{2}^{M}$ \\
\hline Tianjin & 20,856 & 122,680 & 67 \\
Inner Mongolia & 27,375 & 171,743 & 371 \\
Zhejiang & 59,447 & 503,031 & 180 \\
Fujian & 26,560 & 318,267 & 138 \\
Hainan & 2463 & 43,932 & 26 \\
Chongqing & 12,702 & 151,359 & 129 \\
Sichuan & 33,408 & 312,105 & 200 \\
Guizhou & 15,028 & 93,372 & 194 \\
Yunnan & 19,858 & 184,083 & 192 \\
Gansu & 14,352 & 91,036 & 182 \\
\hline
\end{tabular}

Table 5. Input and output improvement after eliminating congestion.

\begin{tabular}{cccccc}
\hline District & $x_{1}^{C}$ & $x_{2}^{C}$ & $g^{C}$ & $u_{1}^{C}$ & $u_{2}^{C}$ \\
\hline Inner Mongolia & 8989 & 2017 & 29,706 & 173,826 & 371 \\
Liaoning & 12,960 & 1900 & 34,780 & 246,612 & 282 \\
Anhui & 9512 & 1361 & 22,798 & 254,329 & 195 \\
Qinghai & 1499 & 465 & 7556 & 54,978 & 44 \\
\hline
\end{tabular}

These congestion districts indicate that rapid expansion of the economy and investment of resources should be conducted with care. The local government must analyze how inefficiencies are caused and propose appropriate measures to improve efficiency, because devoting more resources does not necessarily produce more output. For example, Tianjin can improve its desirable output without changing the input and Qinghai can obtain more desirable outputs while decreasing input.

Table 6 reports the managerial inefficiency and congestion results when only desirable outputs are considered. Column 2 represents output deficiency caused by managerial inefficiency. Columns 3-5 display the output deficiency and input redundancy caused by congestion and the slack variables obtained from Equation (12). Comparing the results of Tables 3 and 6, there is a big difference between managerial inefficiency and congestion. For example, 18 districts reveal undesirable congestion in Table 6 , as compared with four districts in Table 3. This difference shows that if the same amount of input is used to produce more outputs or more types of outputs, then the number of congestion DMUs will be less. According to [6], an occurrence of desirable congestion may be due to technical innovation or undesirable outputs. We may obtain the levels of technical innovation for different districts by comparing their desirable congestion. Therefore, undesirable output is an important factor for performance evaluation during the government's decision-making process. 
Table 6. Managerial inefficiency and congestion, with only desirable output included.

\begin{tabular}{|c|c|c|c|c|}
\hline \multirow{2}{*}{ District } & \multirow{2}{*}{$\begin{array}{c}\begin{array}{c}\text { Managerial } \\
\text { inefficiency }\end{array} \\
g^{M}\end{array}$} & \multicolumn{2}{|c|}{ Input congestion } & \multirow{2}{*}{$\frac{\text { Output congestion }}{s_{1}^{U}}$} \\
\hline & & $s_{1}^{C-}$ & $s_{2}^{C-}$ & \\
\hline Beijing & 0 & 0 & 0 & 0 \\
\hline Tianjin & 1110.89 & 1307.56 & 0 & 2476.04 \\
\hline Hebei & $26,545.83$ & 0 & 0 & 0 \\
\hline Shanxi & $13,005.83$ & 0 & 0 & 0 \\
\hline Inner Mongolia & $21,397.44$ & 0 & 0 & 0 \\
\hline Liaoning & 0 & 6046.14 & 0 & 9310.90 \\
\hline Jilin & 0 & 3611.02 & 0 & 6837.97 \\
\hline Heilongjiang & 8496.04 & 2224.51 & 0 & 5354.40 \\
\hline Shanghai & 0 & 0 & 0 & 0 \\
\hline Jiangsu & 0 & 0 & 0 & 0 \\
\hline Zhejiang & 3798.99 & 0 & 0 & 0 \\
\hline Anhui & 4627.53 & 4425.40 & 0 & 8534.67 \\
\hline Fujian & $16,968.91$ & 990.53 & 0 & 900.87 \\
\hline Jiangxi & 7842.29 & 3273.22 & 0 & 8583.74 \\
\hline Shangdong & 0.00 & 2861.43 & 0 & 1575.80 \\
\hline Henan & $20,119.40$ & 2170.10 & 0 & 1591.81 \\
\hline Hubei & 6140.00 & 3392.50 & 0 & 5156.82 \\
\hline Hunan & $12,982.56$ & 3032.56 & 0 & 4953.39 \\
\hline Guangdong & 0 & 0 & 0 & 0 \\
\hline Guangxi & $20,062.85$ & 764.35 & 0 & 1003.30 \\
\hline Hainan & 4081.66 & 205.45 & 0 & 389.06 \\
\hline Chongqing & 7362.38 & 2587.87 & 0 & 5115.59 \\
\hline Sichuan & 8463.09 & 3069.68 & 0 & 4220.68 \\
\hline Guizhou & $19,104.34$ & 0 & 0 & 0 \\
\hline Yunnan & $20,098.14$ & 0 & 0 & 0 \\
\hline Tibet & 0 & 0 & 0 & 0 \\
\hline Shaanxi & 4467.23 & 2915.79 & 0 & 6459.37 \\
\hline Gansu & $16,876.05$ & 0 & 0 & 0 \\
\hline Qinghai & 0 & 0 & 200.09 & 5121.54 \\
\hline Ningxia & 0 & 0 & 249.15 & 6396.19 \\
\hline Xinjiang & $18,732.48$ & 0 & 0 & 0 \\
\hline
\end{tabular}

It is worth noting that technical inefficiency does not occur in our case. The possible reason lies in the fact that the DMUs consume two inputs to produce one desirable output and two undesirable outputs. That is, when multiple inputs and multiple outputs are taken into consideration, technical inefficiency may not exist. However, this does not affect our analysis of technical inefficiency in this study. Since technical inefficiency can always occur in real applications, we have the incentive to adjust the input and output bundle so as to identify such inefficiency on specific input(s) and output(s). 


\section{Conclusions}

In recent decades, the economy of China has witnessed rapid development, which causes serious environmental problems that arouse widespread concern in society. This paper studies the difference between technical inefficiency and congestion based on definitions from research and real practice. Based on a review of previous literature, we classify congestion as defined by [9] into two parts, technical inefficiency and congestion, and propose mathematical expressions for technical inefficiency, managerial inefficiency, and congestion. We then propose several modified models to identify various inefficiencies. The models are extended by incorporating desirable and undesirable congestion simultaneously. Our methodology can contribute to a better trade-off between economic development and environmental protection.

There are three main contributions of this study. First, congestion is defined well by separating conventional congestion into a new defined congestion and technical inefficiency, which has been defined by other research and accords with production practice. Although technical inefficiency may be smaller in magnitude and may not occur when there are multiple inputs and outputs, it is nontrivial in real applications because of its significant difference from congestion.

Second, we extend the understanding of congestion by positing a situation in which desirable and undesirable congestion are taken into consideration simultaneously. Although several studies have discussed this issue, their identification is based on an inappropriate classification of congestion and will result in a misunderstanding of congestion. From this perspective, we propose desirable and undesirable congestion based on the differentiation of technical inefficiency from conventional congestion and explore modified models to better identify them.

Thirdly, the proposed approach can be verified by applying it to the identification of congestion in China's economic development. We find that several districts exhibit managerial inefficiency and congestion. To solve this problem, we apply the proposed method to these districts to eliminate their managerial inefficiency and congestion. We also find that if the same amount of inputs produces a larger amount of outputs, then the number of congestion DMUs will be less. All these results can be used by the government to promote the development of the economy.

Future research into this approach will involve several aspects. We can deal with resource allocation under centralized organizations, considering congestions in the DEA framework. The opportunities present for research into possibilities for reallocating congesting inputs between different DMUs will result in output increase. Other possibilities for research include considering the relationship between desirable outputs and undesirable outputs, where an increase in undesirable outputs decreases the vector of desirable outputs.

\section{Acknowledgments}

This work was supported by the National Natural Science Foundation of China (Grant Nos. 71371008 and 71001093), Major International (Regional) Joint Research Projects (Grant No. 71110107024)

and the National Natural Science Foundation of China for Innovative Research Groups (Grant No. 71121061). 


\section{Author Contributions}

Jun Wang established the model and analyzed the results. Yong Zha gave the framework of this research. We drafted the article together.

\section{Appendix}

The original data were collected from the China Statistical Yearbook 2013 [30] and are shown in Table A1.

Table A1. The description of original data.

\begin{tabular}{|c|c|c|c|c|c|}
\hline District & $x_{1}(100$ million $)$ & $x_{2}(100$ million kwh $)$ & $y$ (100 million yuan) & $u_{1}(10000 \mathrm{t})$ & $u_{2}(10000 t)$ \\
\hline Beijing & 4992.93 & 874.28 & $28,613.16$ & $140,273.72$ & 33.82 \\
\hline Tianjin & 5481.30 & 722.48 & $19,986.14$ & $82,813.16$ & 64.28 \\
\hline Hebei & $15,675.60$ & 3077.73 & $33,567.18$ & $305,773.50$ & 433.82 \\
\hline Shanxi & 7052.39 & 1765.79 & $25,342.08$ & $134,298.48$ & 361.66 \\
\hline Inner Mongolia & 9166.39 & 2016.76 & $21,754.23$ & $102,424.49$ & 363.68 \\
\hline Liaoning & $16,632.28$ & 1899.88 & $34,779.77$ & $238,768.79$ & 282.13 \\
\hline Jilin & 7323.47 & 637.00 & $13,896.98$ & $119,509.20$ & 124.41 \\
\hline Heilongjiang & 6967.20 & 827.91 & $13,223.14$ & $162,589.08$ & 199.42 \\
\hline Shanghai & 4230.00 & 1353.45 & $31,160.89$ & $219,244.06$ & 71.70 \\
\hline Jiangsu & $25,207.36$ & 4580.90 & $84,550.41$ & $598,210.95$ & 291.48 \\
\hline Zhejiang & $14,372.15$ & 3210.55 & $55,654.17$ & $420,960.52$ & 168.86 \\
\hline Anhui & $12,073.25$ & 1361.10 & $22,797.65$ & $254,328.89$ & 190.29 \\
\hline Fujian & 9829.45 & 1579.50 & $21,385.98$ & $256,262.77$ & 109.11 \\
\hline Jiangxi & 8230.48 & 867.67 & $11,967.66$ & $201,189.71$ & 150.22 \\
\hline Shandong & $23,780.35$ & 3794.55 & $71,107.66$ & $479,100.25$ & 418.30 \\
\hline Henan & $17,380.19$ & 2747.75 & $35,174.81$ & $403,667.62$ & 350.16 \\
\hline Hubei & $11,840.67$ & 1507.85 & $26,877.66$ & $290,200.42$ & 161.20 \\
\hline Hunan & $10,593.81$ & 1345.22 & $17,784.25$ & $304,214.43$ & 159.29 \\
\hline Guangdong & $14,923.40$ & 4619.41 & $71,343.84$ & $838,550.53$ & 243.09 \\
\hline Guangxi & 7279.60 & 1153.42 & $11,759.56$ & $245,577.80$ & 130.21 \\
\hline Hainan & 1603.23 & 208.08 & 2023.16 & $37,103.42$ & 15.41 \\
\hline Chongqing & 6764.58 & 723.03 & $11,113.36$ & $132,430.20$ & 112.97 \\
\hline Sichuan & $13,278.54$ & 1830.70 & $30,362.89$ & $283,657.06$ & 181.93 \\
\hline Guizhou & 4186.20 & 1046.72 & 8302.29 & $91,455.12$ & 189.91 \\
\hline Yunnan & 5832.03 & 1315.86 & $13,076.97$ & $154,009.74$ & 160.72 \\
\hline Tibet & 424.68 & 27.76 & 506.86 & 4683.14 & 5.51 \\
\hline Shaanxi & 8958.37 & 1066.75 & 20591.16 & $128,749.05$ & 211.39 \\
\hline Gansu & 3991.75 & 994.56 & 9146.01 & $62,813.02$ & 125.34 \\
\hline Qinghai & 1499.29 & 602.22 & 4041.92 & $21,994.46$ & 43.63 \\
\hline Ningxia & 1759.10 & 741.79 & 4860.19 & $38,948.08$ & 106.04 \\
\hline Xinjiang & 5092.06 & 1090.80 & $11,669.17$ & $93,810.49$ & 231.17 \\
\hline
\end{tabular}

\section{Conflicts of Interest}

The authors declare no conflict of interest. 


\section{References}

1. Wang, K.; Zhang, X.; Wei, Y.-M.; Yu, S. Regional allocation of $\mathrm{CO}_{2}$ emissions allowance over provinces in China by 2020. Energy Policy 2013, 54, 214-229.

2. Cooper, W.W.; Deng, H.; Gu, B.; Li, S.; Thrall, R. Using DEA to improve the management of congestion in Chinese industries (1981-1997). Soc. Econ. Plan. Sci. 2001, 35, 227-242.

3. Färe, R.; Grosskopf, S. Measuring congestion in production. J. Econ. 1983, 43, 257-271.

4. Sueyoshi, T. DEA implications of congestion. Asia Pac. Manag. Rev. 2003, 8, 59-70.

5. Korhonen, P.J.; Luptacik, M. Eco-efficiency analysis of power plants: An extension of data envelopment analysis. Eur. J. Oper. Res. 2004, 154, 437-446.

6. Sueyoshi, T.; Goto, M. Weak and strong disposability vs. natural and managerial disposability in DEA environmental assessment: Comparison between Japanese electric power industry and manufacturing industries. Energy Econ. 2012, 34, 686-699.

7. Wu, J.; An, Q.; Xiong, B.; Chen, Y. Congestion measurement for regional industries in China: A data envelopment analysis approach with undesirable outputs. Energy Policy 2013, 57, 7-13.

8. Wei, Q.; Yan, H. Congestion and returns to scale in data envelopment analysis. Eur. J. Oper. Res. 2004, 153, 641-660.

9. Tone, K.; Sahoo, B.K. Degree of scale economies and congestion: A unified DEA approach. Eur. J. Oper. Res. 2004, 158, 755-772.

10. Brockett, P.; Cooper, W.; Deng, H.; Golden, L.; Ruefli, T. Using DEA to identify and manage congestion. J. Prod. Anal. 2004, 22, 207-226.

11. Färe, R.; Svensson, L. Congestion of production factors. Econom. J. Econom. Soc. 1980, 48, 1745-1753.

12. Färe, R.; Grosskopf, S. Slacks and congestion: A comment. Soc. Econ. Plan. Sci. 2000, 34, $27-33$.

13. Cooper, W.W.; Deng, H.; Huang, Z.M.; Li, S.X. A one-model approach to congestion in data envelopment analysis. Soc. Econ. Plan. Sci. 2002, 36, 231-238.

14. Flegg, A.; Allen, D.O. Does expansion cause congestion? The case of the older British universities, 1994-2004. Educ. Econ. 2007, 15, 75-102.

15. Simões, P.; Marques, R.C. Influence of Congestion Efficiency on the European Seaports Performance: Does It Matter? Transp. Rev. 2010, 30, 517-539.

16. Khodabakhshi, M. A one-model approach based on relaxed combinations of inputs for evaluating input congestion in DEA. J. Comput. Appl. Math. 2009, 230, 443-450.

17. Noura, A.; Hosseinzadeh Lotfi, F.; Jahanshahloo, G.; Rashidi, S.F.; Parker, B.R. A new method for measuring congestion in data envelopment analysis. Soc. Econ. Plan. Sci. 2010, 44, 240-246.

18. Khoveyni, M.; Eslami, R.; Khodabakhshi, M.; Jahanshahloo, G.R.; Hosseinzadeh Lotfi, F. Recognizing strong and weak congestion slack based in data envelopment analysis. Comput. Ind. Eng. 2013, 64, 731-738.

19. Kao, C. Congestion measurement and elimination under the framework of data envelopment analysis. Int. J. Prod. Econ. 2010, 123, 257-265.

20. Simões, P.; Marques, R.C. Performance and congestion analysis of the portuguese hospital services. Cent. Eur. J. Oper. Res. 2011, 19, 39-63. 
21. Marques, R.C.; Simões, P. Measuring the influence of congestion on efficiency in worldwide airports. J. Air Transp. Manag. 2010, 16, 334-336.

22. Färe, R.; Grosskopf, S.; Lovell, C.K.; Pasurka, C. Multilateral productivity comparisons when some outputs are undesirable: A nonparametric approach. Rev. Econ. Stat. 1989, 71, 90-98.

23. Hailu, A.; Veeman, T.S. Non-parametric productivity analysis with undesirable outputs: An application to the Canadian pulp and paper industry. Am. J. Agric. Econ. 2001, 83, 605-616.

24. Seiford, L.M.; Zhu, J. Modeling undesirable factors in efficiency evaluation. Eur. J. Oper. Res. 2002, 142, 16-20.

25. Ji, J.; Wang, Y. Commercial Bank Efficiency Evaluation in Consideration of the Undesirable Output and Its Link with Stakeholders Relationship: An Application of China's Commercial Banks. Math. Probl. Eng. 2014, 2014, Article 949717.

26. Jahanshahloo, G.R.; Lotfi, F.H.; Shoja, N.; Tohidi, G.; Razavyan, S. Undesirable inputs and outputs in DEA models. Appl. Math. Comput. 2005, 169, 917-925.

27. Huang, J.; Chen, J.; Yin, Z. A Network DEA Model with Super Efficiency and Undesirable Outputs: An Application to Bank Efficiency in China. Math. Probl. Eng. 2014, 2014, Article 793192.

28. Li, J.; Li, J.; Zheng, F. Unified Efficiency Measurement of Electric Power Supply Companies in China. Sustainability 2014, 6, 779-793.

29. Banker, R.D.; Charnes, A.; Cooper, W.W. Some models for estimating technical and scale inefficiencies in data envelopment analysis. Manag. Sci. 1984, 30, 1078-1092.

30. National Bureau of Statistics. China Statistical Yearbook 2013; National Bureau of Statistics: Beijing, China, 2014. (In Chinese)

(C) 2014 by the authors; licensee MDPI, Basel, Switzerland. This article is an open access article distributed under the terms and conditions of the Creative Commons Attribution license (http://creativecommons.org/licenses/by/4.0/). 